\title{
Gravitational waves from inspiraling compact binaries: Second post-Newtonian waveforms as search templates II
}

\author{
Serge Droz \\ Department of Physics, University of Guelph, Guelph, Ontario, N1G 2W1, Canada
}

\begin{abstract}
We present further evidence that the second post-Newtonian $(\mathrm{pN})$ approximation to the gravitational waves emitted by inspiraling compact binaries is sufficient for the detection of these systems. This is established by comparing the 2 - $\mathrm{pN}$ wave forms to signals calculated from black hole perturbation theory. Results are presented for different detector noise curves. We also discuss the validity of this type of analysis.
\end{abstract}

Pacs numbers: 04.25.Nx, 04.30.Db, 04.80.Nn

\section{INTRODUCTION}

There is currently a large effort underway to build an international network of interferometric gravitational wave detectors. These include the two American LIGO detectors, the French-Italian VIRGO detector, the somewhat smaller British-German GEO600 and the Japanese TAMA detector.

One of the most promising sources for detection are compact inspiraling binary systems [1]. These systems, consisting of a pair of black holes or neutron stars, lose energy through the emission of gravitational radiation and therefore spiral inward. The emission is strongest during the last few minutes of the inspiral; just before the orbit becomes unstable and the two objects plunge towards each other. For stars with masses a few times that of the sun this last burst of waves falls within the frequency band of the previously mentioned detectors.

The method of choice to search for such signals in a noisy data stream is matched filtering [2], where the detector output is compared to a bank of templates. A good overlap or match then indicates the presence of a signal. It can be shown [2] that matched filtering is an optimal method. However, to use this technique an accurate knowledge of the expected signals is necessary to set up the template bank. Since no exact solutions of Einstein's equations are available which describe a binary inspiral, we have to resort to some approximation scheme. The templates used by LIGO are calculated using a post Newtonian $(\mathrm{pN})$ expansion in the orbital velocity of the binary 3 - 5]. However, since the $\mathrm{pN}$ expansion converges slowly [6], if at all, there is a need to ascertain the quality of these templates.

How does one assess the quality of these templates? In a first paper [7] we compared the 2-pN templates to waveforms calculated from black hole perturbation theory $[8,9$. It was found that the $2-\mathrm{pN}$ templates are sufficiently accurate for the detection of a binary signal, but not for parameter estimation. However, this first paper did not elaborate on the validity of the analysis presented. In this publication we address this question, and also present results for a wider selection of detector noise curves.

The paper is organized as follows. In section [1] we introduce the notation and briefly present the formalism used in the remainder of the paper. Specifically we introduce the fitting factor [10] which is a measure of the quality of a template set. In section [II] we address some of the problems encountered when calculating the fitting factor using black hole perturbation theory. Finally, in section IV we present our results, and finally in section \ we summarize our conclusions.

\section{ASSESSING THE QUALITY OF A TEMPLATE SET}

\section{A. The fitting factor}

Let us consider the output of a gravitational wave detector of the form

$$
s(t)=h(t, \vec{\mu})+n(t) .
$$

Here $n(t)$ denotes the detector noise and $h(t, \vec{\mu})$ is the (possibly absent) signal, which depends on a set of parameters $\vec{\mu}$. We define the signal to noise ratio (SNR) 111] associated with the template $t_{i}$ by

$$
\rho_{i}=\frac{\left(s \mid t_{i}\right)}{\operatorname{rms}\left(n \mid t_{i}\right)},
$$

where the inner product $(\cdot \mid \cdot)$ is defined by

$$
(s \mid t):=2 \int_{0}^{\infty} \frac{d f}{S_{n}(f)}\left(\hat{s}(f) \hat{t}^{*}(f)+\hat{s}^{*}(f) \hat{t}(f)\right) .
$$

As usual hats denote the Fourier transform $\hat{s}(f)=$ $\int e^{-2 \pi i f t} s(t) d t$ and an asterix indicates complex conjugation. The function $S_{n}(f)$ is the noise's one sided spectral density.

In this work we use an analytic fit to the noise curves of the various detectors 12, 13.

$$
S_{n}(f)=S_{0}\left(S_{1}+4\left(\frac{f}{f_{\mathrm{m}}}\right)^{2}+2\left(\frac{f}{f_{\mathrm{m}}}\right)^{-4}+2\left(\frac{f}{f_{1}}\right)^{-18}\right)
$$




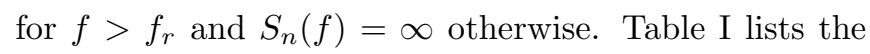
parameters for a few different detectors.

\begin{tabular}{lccccc}
\hline \hline Detector & $S_{0}$ & $S_{1}\left[\mathrm{~Hz}^{-1}\right]$ & $f_{\mathrm{m}}[\mathrm{Hz}]$ & $f_{1}[\mathrm{~Hz}]$ & $f_{r}[\mathrm{~Hz}]$ \\
\hline Caltech 40m & $0.5 \times 10^{-42}$ & 10.0 & 610.0 & 183.0 & 140.0 \\
initial LIGO & $0.5 \times 10^{-46}$ & 4.0 & 200.0 & 0.0 & 40.0 \\
advanced LIGO & $1.5 \times 10^{-49}$ & 4.0 & 70.0 & 0.0 & 10.0 \\
\hline
\end{tabular}

TABLE I. The parameters used in the analytic fit of the various detector noise curves. Note that the value of $S_{0}$ is not important for the calculation of the fitting factor.

For Gaussian noise [11 the expectation value of the SNR is given by

$$
\left\langle\rho_{i}\right\rangle=\frac{\left(h \mid t_{i}\right)}{\sqrt{\left(t_{i} \mid t_{i}\right)}} .
$$

This expression is maximized for $t_{i}=h$, i.e. for the Wiener optimal filter. We can thus rewrite equation (5) as

$$
\left\langle\rho_{i}\right\rangle=\frac{\left(h \mid t_{i}\right)}{\sqrt{\left(t_{i} \mid t_{i}\right)(h \mid h)}}\langle\rho\rangle_{\max } .
$$

The quantity

$$
\mathcal{A}_{i}=\frac{\left(h \mid t_{i}\right)}{\sqrt{\left(t_{i} \mid t_{i}\right)(h \mid h)}}
$$

is called the ambiguity function. It gives the loss of SNR due to an inaccurate template $t_{i}$. Its maximum $F F=$ $\max _{i} \mathcal{A}_{i}$ is the fitting factor 10. It gives the loss of SNR due to a non optimal set of templates.

Obviously to calculate the fitting factor we need to know the signal $h$ we are looking for. In this paper we use a signal calculated from black hole perturbation theory as a substitute for the "real" signal $h[8]$. Black hole perturbation theory is a priori only applicable in the small mass ratio limit. However, we believe that black hole perturbation theory can give useful information for larger mass ratios. We elaborate on this statement below.

Let us now discuss the signals and templates in some detail.

\section{B. The reference signal}

Black hole perturbation theory [8] gives the two independent components of the gravitational wave field as

$h_{+}(t)-i h_{\times}(t) \propto \sum_{l=2}^{\infty} \sum_{m=-l}^{l=m} A_{l m}(v) e^{i m \Omega t}{ }_{-2} Y_{l m}(\vartheta, \varphi)$.

Here the functions $A_{l m}(v)$ are the mode amplitudes which are calculated numerically by solving the Teukolsky equation. The functions ${ }_{-2} Y_{l m}(\vartheta, \varphi)$ are spinweighted spherical harmonics [14]; the angles $\vartheta$ and $\varphi$ refer to a Cartesian system centered at the black hole with the $z$-axis orthogonal to the orbital plane. The orbital velocity $v$ is related to the angular velocity $\Omega$ by the relation $v^{3}=M \Omega$, where $M=m_{1}+m_{2}$ is the total mass.

This waveform describes a particle moving in a fixed circular orbit around a Schwarzschild black hole. We can easily generalize this expression to an adiabatic inspiral (see Ref. [7] for details). To simplify expression (7) we choose $\varphi=0$ (since the orbit is circular this represents no loss of generality) and a detector which singles out the + polarization. Finally, we calculate the Fourier transform by means of a stationary phase approximation. We obtain

$$
\hat{h}(f) \propto \sum_{l=2}^{\infty} \sum_{m=2}^{l=m} B_{l m}(v) e^{i m \psi(v)} S_{l m}(\vartheta) .
$$

The functions $B_{l m}(v)$ and $\psi(v)$ are calculated numerically (see Ref. [7] for details) and $S_{l m}(\vartheta)={ }_{-2} Y_{l m}(\vartheta, 0)+$ $(-1)_{-2}^{l} Y_{l-m}(\vartheta, 0)$. In this expression the gravitational wave frequency is related to the orbital velocity by $f=$ $m v^{3} / 2 \pi M$. Black hole perturbation theory predicts that the innermost stable circular orbit (ISCO) is located at $v_{\mathrm{ISCO}}=1 / \sqrt{6}$, after which $h(v) \equiv 0$. In principle, the sum over the index $l$ extends to infinity. We have numerically verified that in practice we can truncate the series at $l=3$.

\section{The templates}

As was mentioned previously, the templates to be used for LIGO data analysis are the restricted 2-pN waveforms [3]. (The term restricted means that we keep the phase accurate to second $\mathrm{pN}$ order but use only the leading order amplitude.) Using the stationary phase approximation gives

$$
\hat{t_{i}}:=\hat{t}(f, \vec{\theta}) \propto f^{-\frac{7}{6}} e^{i \psi(f)},
$$

where $\psi(f)=2 \pi f t_{c}-\phi_{c}+\phi(f)$ and

$$
\begin{aligned}
\phi(f)= & \frac{3}{128}(\pi \mathcal{M} f)^{-5 / 3}\left[1+\frac{20}{9}\left(\frac{743}{336}+\frac{11}{4} \eta\right) x^{2}-16 \pi x^{3}\right. \\
& \left.+10\left(\frac{3058673}{1016064}+\frac{5429}{1008} \eta+\frac{617}{144} \eta^{2}\right) x^{4}\right],
\end{aligned}
$$

with $x^{3}=\pi \eta^{-3 / 5} \mathcal{M} f$. Here, $\vec{\theta}=\left\{\phi_{c}, t_{c}, \mathcal{M}, \eta\right\}$ are the template parameters, with $\phi_{c}$ the phase at coalescence (formally $f=\infty$ ), $t_{c}$ the time at coalescence, $\mathcal{M}=\left(m_{1} m_{2}\right)^{\frac{3}{5}} M^{-\frac{1}{5}}$ the chirp mass, and $\eta=m_{1} m_{2} / M^{2}$ the mass-ratio parameter. Note that $\eta$ is maximal for for $m_{1}=m_{2}$ with a value of 0.25 . 


\section{VALIDITY OF THE TEST MASS APPROXIMATION}

Black hole perturbation theory is valid only for small values of $\eta$, say $\eta<\eta_{\max }=0.1$. This poses a problem, since most systems we are interested in have a mass ratio of $\eta>0.2$. Furthermore, for a fixed chirp mass, binaries with small mass ratios are more relativistic and we expect the $\mathrm{pN}$ templates to perform poorly.

We can estimate of the minimal value that $\eta$ should take for the $\mathrm{pN}$ approximation to be valid. We expect $\mathrm{pN}$ corrections in the phase (10) to be small if $x<x_{0}=0.25$. Then, for fixed chirpmass $\mathcal{M}$ (i.e. for a fixed number of wave cycles), we find

$$
\eta_{\min }=x_{0}^{-5}(\pi f \mathcal{M})^{\frac{5}{3}}
$$

at a given frequency $f$. For example, for $\mathcal{M}=1 M_{\odot}$, $f=200 \mathrm{~Hz}$ (the peak sensitivity of the initial LIGO detector) and $x_{0}=0.25$ we get $\eta_{\min }=0.06$. Thus for $\eta \in[0.06,0.1]$ we expect out analysis to give valid results.

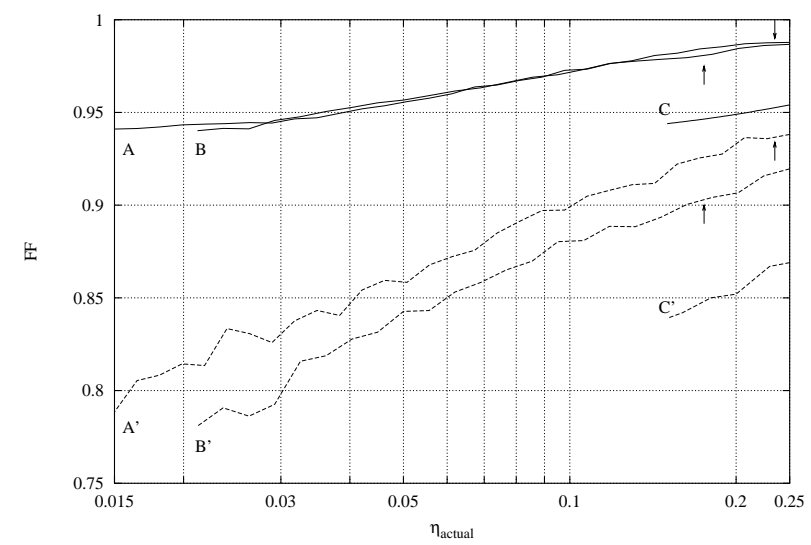

FIG. 1. shows the fitting factor as a function of $\eta_{\text {actual }}$ (i.e. the signal's $\eta$ ) for constant values of the chirp mass $\mathcal{M}$ for the 40 meter noise curve. The three systems shown in this plot correspond to chirp masses $\mathcal{M}_{A}=0.87 M_{\odot}$, $\mathcal{M}_{B}=1.22 M_{\odot}$ and $\mathcal{M}_{C}=3.92 M_{\odot}$. The primed labels correspond to signals which include all modes $l=2,3,|m|<l$, whereas the unprimed labels correspond to signals only consisting of the (dominant) $l=m=2$ mode. The arrows indicate where the respective curves leave the targeted mass range $0.8 M_{\odot}<m<3.2 M_{\odot}$ of the detector.

However we still cannot say anything about systems with mass ratios larger than $\eta>0.1$. Furthermore, for some noise curves $\eta_{\min }>\eta_{\max }$. For example, at the peak sensitivity of the 40 -meter noise curve $(f=610 \mathrm{~Hz})$ we get $\eta_{\min }>0.25$.

To estimate how important the corrections to the fitting factor due to non-zero $\eta$ are, we can set $\eta=0$ in the coefficients of $x$ in the phase (10). This essentially gives us a 2-pN approximation to the perturbation theory signal. Somewhat surprisingly we find, within numerical accuracy, no difference in the fitting factors calculated for the two types of templates. This strongly suggests that the non-zero corrections in (10) play no essential role, and that our analysis stays valid even for $\eta>\eta_{\max }$.

Since the binary system becomes less relativistic with increasing $\eta$ and constant chirp mass we expect the fitting factor to increase as well. This provides us with an additional consistency check. This behaviour is indeed observed (see Figure 11).

\section{RESULTS}

Calculating the fitting factor is in principle straightforward. However evaluating the inner product (3) numerically can be tricky. Two methods were used to numerically calculate the fitting factor. In the first we calculated the integral in (3) using a Romberg integration [15]. The second method uses the fact that $\hat{h}(f) \propto \exp 2 \pi t_{c} f$ and thus the overlap integral (3) can be evaluated using FFTs. Signal and templates in the latter case were calculated using the GRASP library [16]. For all relevant cases the two methods give the same answer within a few percent. As it turns out, the second method is a lot faster despite the many more " $t_{c}$ " values that are calculated.

The ambiguity function depends a priory on the four parameters $\phi_{c}, t_{c}, \mathcal{M}$ and $\eta$. Maximization over the phase $\phi_{c}$ is trivial. If FFTs are used maximization over $t_{c}$ is also $\mathrm{s}$ trivial. This leaves us with a reduced ambiguity function to maximize, depending on three or two variables, respectively. In the first case maximization is difficult, because $\mathcal{A}\left(t_{c}, \mathcal{M}, \eta\right)$ possesses many local maxima. These seem to mostly disappear after maximization over $t_{c}$, so maximizing the ambiguity function using FFTs is much easier.

In a first run, reported in [7], we calculated fitting factors for the restricted $2 \mathrm{pN}$ templates assuming the advanced LIGO noise curve. We found that the templates are generally adequate for detection, but not for parameter estimation. We now have extended the analysis to also cover the Caltech 40 meter prototype and the initial LIGO noise curves. Our results are summarized in Table II for a few selected cases. For each detector there is at least one system with a fitting factor larger than $90 \%$ which is deemed acceptable by Apostolatos [10].

\begin{tabular}{ccccc}
\hline \hline & \multicolumn{2}{c}{ Advanced LIGO } & initial LIGO & 40-meter \\
Templates & Newtonian & $2 \mathrm{pN}$ & $2 \mathrm{pN}$ & $2 \mathrm{pN}$ \\
\hline $1.4-1.4 M_{\odot}$ & $97.2 \%$ & $93.0 \%$ & $95.8 \%$ & $94.0 \%$ \\
$0.5-5.0 M_{\odot}$ & $51.6 \%$ & $95.2 \%$ & $89.7 \%$ & $89.0 \%$ \\
$1.4-10.0 M_{\odot}$ & $55.8 \%$ & $91.4 \%$ & $88.4 \%$ & $<85.0 \%$ \\
$10.0-10.0 M_{\odot}$ & $70.1 \%$ & $91.7 \%$ & $86.3 \%$ & \\
$4.0-30.0 M_{\odot}$ & $61.3 \%$ & $86.6 \%$ & $67.9 \%$ & \\
\hline \hline
\end{tabular}

TABLE II. The fitting factors $F F$ for selected binary systems and for various noise curves. 
A more complete picture is given by Figure 2 which shows the contours of $F F=90 \%$ in the $m_{1}-m_{2}$ plane for the 40 meter, initial and advanced LIGO detectors. Since the frequency band for the advanced LIGO starts at $10 \mathrm{~Hz}$ we have to follow a large number of wave cycles, especially for low mass systems. This makes the evaluation of the fitting factor difficult in terms of available computer power.

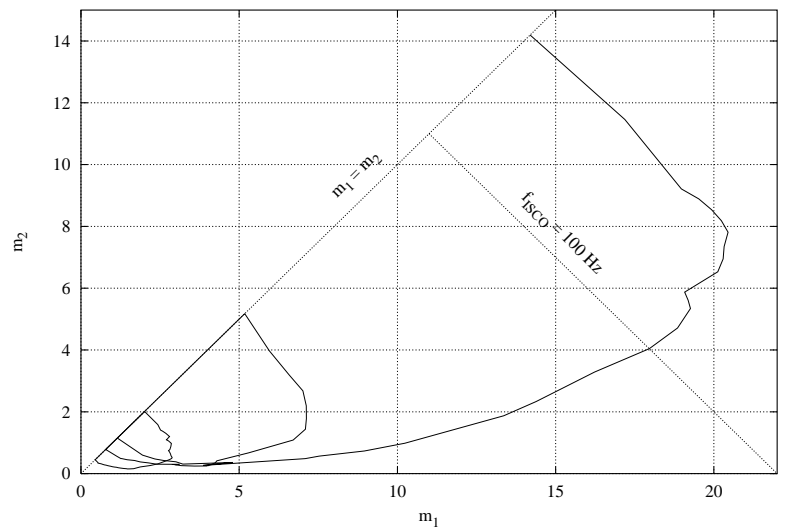

FIG. 2. shows the contours of $F F=90 \%$ in the $m_{1}-m_{2}$ plane for the Caltech 40 meter prototype, the initial and advanced LIGO detectors. Units are in solar masses. The dotted curve to the right denotes the line where the frequency at the inner most stable orbit (ISCO), as predicted by perturbation theory, becomes less than $100 \mathrm{~Hz}$. While there are still templates with $F F>90 \%$ in this region, the total SNR for these systems is very low since they spend very little time in the advanced LIGO frequency band.

In a next step we calculated the fitting factor as function of $\eta$ for fixed chirp mass $\mathcal{M}$. (These are the mass parameters for the signal and they should not be confused with the mass parameters in the ambiguity function $\mathcal{A}$.) As expected, the fitting factor drops considerably as $\eta$ decreases. Figure 1 shows the curves $F F(\eta)$ for three different values of the chirp mass for the 40 meter noise curve. Note that the end points of these curves lie well outside the mass regime considered of interest for this detector. Similar results were obtained for the advanced and initial LIGO detectors.

Finally, we repeated this calculation with $\eta=0$ in the coefficients of the phase (10). The values for the fitting factor were found to agree with the previous values within numerical accuracy. Figure 3 compares the two cases for the $40 \mathrm{~m}$ noise curve for the $l=m=2$ signal. We take this as an indication that perturbation theory waveforms are adequate to test the performance of a set of templates, even when $\eta$ is not small.

\section{CONCLUSIONS}

The results of this analysis suggest that the $2 \mathrm{pN}$ templates are adequate for the detection of gravitational ra-

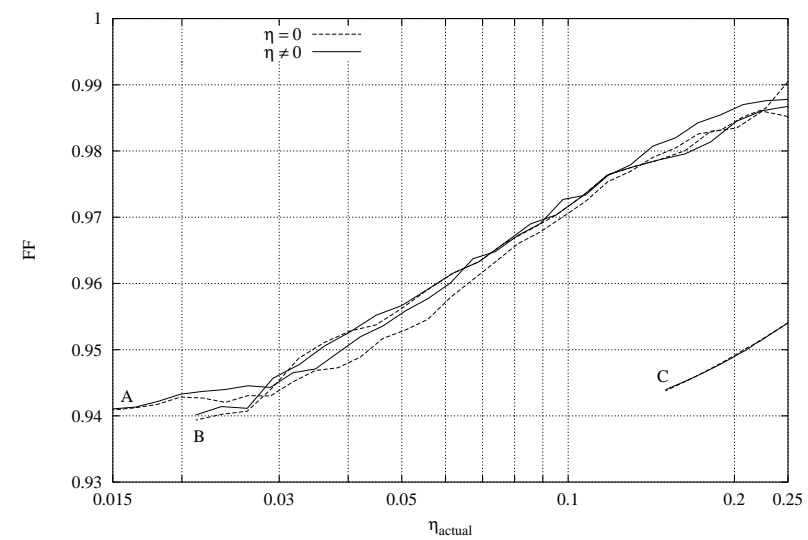

FIG. 3. shows plots of the fitting factor $F F$ as a function of $\eta$ with constant chirp mass $\mathcal{M}$ for the same three systems. The curves shown were calculated using test mass signals consisting of the $l=m=2$ mode only. The dashed curves correspond to templates with $\eta=0$. The solid lines represent proper $2 \mathrm{pN}$ templates. There is practically no difference between the two cases, indicating that perturbation theory gives valid results even for relatively large mass ratios.

diation emitted from inspiraling compact binary systems, when the masses lie in the region shown in Figure 2. We emphasize that we do not claim that the $2 \mathrm{pN}$ templates are all we need to know for a LIGO-type search for compact binaries. Clearly, more accurate templates are needed to detect the larger mass systems as well as for parameter estimation [7]. But we do believe that the 2-pN templates give us a good chance to detect gravitational wave from, at least, some systems.

While it is not a priory clear that black hole perturbation theory makes a good tool to ascertain the quality of 2-pN templates for large mass ratio systems, we have given arguments to support our results. At the very least, we feel our analysis gives a qualitatively correct picture.

\section{ACKNOWLEDGMENTS}

The authour would like to thank Eric Poisson for his stimulating comments. This work was supported by the Natural Sciences and Engineering Research Council of Canada.

[1] K. S. Thorne, Gravitational radiation, in Three hundred years of gravitation, edited by S. W. Hawking and W. Israel, chap. 9, pp. 330-458, Cambridge University Press, Cambridge, 1987.

[2] L. A. Wainstein and V. D. Zubakov, (Prentice-Hall, London, 1962). 
[3] L. Blanchet, B. R. Iyer, C. M. Will, and A. G. Wiseman, Class. Quant. Grav. 13, 575 (1996), gr-qc/9602024.

[4] C. M. Will and A. G. Wiseman, Phys. Rev. D54, 4813 (1996), gr-qc/9608012.

[5] L. Blanchet, T. Damour, and B. R. Iyer, Phys. Rev. D51, 5360 (1995), gr-qc/9501029.

[6] E. Poisson, Phys. Rev. D52, 5719 (1995), gr-qc/9505030.

[7] S. Droz and E. Poisson, Phys. Rev. D56, 4449 (1997), gr-qc/9705034.

[8] E. Poisson, Phys. Rev. D47, 1497 (1993).

[9] S. A. Teukolsky, APJ 185, 635 (1973).

[10] T. A. Apostolatos, Phys. Rev. D54, 2421 (1996).

[11] C. Cutler and E. E. Flanagan, Phys. Rev. D49, 2658 (1994), gr-qc/9402014.

[12] A. Abramovici et al., Science 256, 325 (1992).

[13] E. E. Flanagan and S. A. Hughes, Phys. Rev. D57, 4535 (1998), gr-qc/9701039.

[14] J. Goldberg, A. MacFarlane, E. Newman, F. Rohrlich, and E. Sudarshan, J. Math. Phys. 8, 2155 (1967).

[15] W. A. Press, S. A. Teukolsky, W. T. Vetterling, and B. P. Flannery, Numerical Recipes: The Art of Scientific Computing (Cambridge University Press, Cambridge, 1995).

[16] B. Allen, GRASP: a data analysis package for gravitational wave detection (LIGO Collaboration, 1997), http://www.ligo.caltech.edu/LIGO_web/Collaboration/manual.pdf. 